Research Article

\title{
Motion Modeling and Control of Lower Limb Exoskeleton Based on Max-Plus Algebra
}

\author{
Cuifeng Qin (iD) and Zuojun Liu (iD) \\ School of Artificial Intelligence, Hebei University of Technology, Tianjin 300130, China \\ Correspondence should be addressed to Zuojun Liu; liuzuojun@hebut.edu.cn
}

Received 28 May 2021; Accepted 13 October 2021; Published 1 November 2021

Academic Editor: Shiping Wen

Copyright (C) 2021 Cuifeng Qin and Zuojun Liu. This is an open access article distributed under the Creative Commons Attribution License, which permits unrestricted use, distribution, and reproduction in any medium, provided the original work is properly cited.

\begin{abstract}
Max-plus algebra is a special method to describe the discrete event system. In this paper, it is introduced to describe the motion of lower limb exoskeleton. Based on the max-plus algebra and the timed event graph, the walking process of exoskeleton is modelled. The max-plus algebra approach can describe the logical sequence and safety condition in the walking process, which cannot be achieved via other conventional modelling approaches. The autonomous control of lower limb exoskeleton system is studied via the model based on max-plus algebra. In the end, an FSM (finite state machine) controller embedded with the max-plus algebra model is proposed, and the experiments show ideal speed and gait/phase period control effect, as well as the good safety and stable performance.
\end{abstract}

\section{Introduction}

Exoskeleton robot is a wearable robot that can be used to enhance people's ability, realize the rehabilitation training of patients, and assist people in various operations. Exoskeletons are of interest for the researchers in the field of robotics and automatic control $[1,2]$. In general, there are two kinds of exoskeleton robots, the rehabilitation exoskeleton and the enhancing physical capacity exoskeleton. In this paper, only the lower limb exoskeleton for enhancing the walking capacity is considered.

The mathematic model of a system describes the dynamic characteristics and the relationship between the variables in the system, which is the fundamental of exoskeleton control. The model of exoskeleton and its stability analyses in most literature is based on the conventional Newton Euler method or Lagrange method $[3,4]$. Both methods can only reflect the characteristics of joint angle, speed, driving torque with time, and load. For lower limb exoskeleton, neither of the two methods can describe the motion logical sequence in the process of walking, e.g., if one leg is in the sway phase, the other one must be in the stance phase, or the falling of foot can only occur after the foot lifts, etc. Fortunately, max-plus algebra and timed event graph could represent such logical sequence in the control process [5].

To assure the gait sequence and safety in the walking process of lower limb exoskeleton, the periodic stability must be ensured. Samuel et al proposed an approach of virtual constraint control, in which a velocity-modulated dead zone is used to obtain the robust stability [6]. Ma et al build an assist torque predictor according to a gait phase classifier based on kernel recursive least-squares algorithm [7]. Li et al proposed a human lower limb joint muscular torques estimation method based on the inverse dynamics of the exoskeleton wearer [8]. Considering the periodic walking trajectory and uncertainties of human-exoskeleton system, Li et al proposed a human-cooperative adaptive fuzzy strategy combing virtual tunnels that allows human subjects to change the movement timing of their legs and produce a physiological path [9]. Lim et al proposed a control strategy based on the time delayed feedback by using a simple leg swing model, in which the stability was analysed [10]. Nasiri et al presented an exoskeleton torque optimization method by using a biomechanical model and proved its stability, convergence, optimality, and robustness [11]. All the 
research above mainly focused on the real-time control performance based on conventional dynamics model approaches, such as Newton Euler and Lagrange, or model-free approaches, such as sensor-triggered control methods. The gait uniformity and symmetry are obtained by setting the desired trajectories in the closed control loop. The safety relays on the extra control command according to the danger alarm of special sensor signals [12]. However, the gait safety constraint and motion sequence are not involved in the system model. Although it can work effectively, it is just solved in the technical way, which lacks theory fundamental.

In these literatures, the motion process is modelled in the way of continuous event system. While, in this paper, the analysis and control of lower limb exoskeleton is consider in the way of discrete event system, in which the max-plus algebra is used for modelling. Max-plus algebra is a mathematic tool for the modelling and performance analysis of discrete event system. It is concerned with the sequence of events, where events are viewed as sudden changes in a process. Although max-plus algebra is not a well-known method, it works well in the biological analysis, network coordinating, production management, and traffic planning [12-15]. Lopes et al used max-plus algebra in the modelling and control of multilegged robots [16]. Tao et al analysed the periodic stability of upper limb rehabilitation robot via max-plus algebra [17]. These papers provide fruitful reference for our research. In [18], we analysed the periodic steady state, disturbance estimation of lower limb exoskeleton based on the model of max-plus algebra. However, the work is only in a primary theoretical way. The deep research and experiment in the exoskeleton control is not involved, which would be presented in this paper.

By using the max-plus algebra method, this paper aims to improve the control performance in terms of safety of lower limb exoskeleton. It would provide a new research thought for the safety and gait/phase control of lower limb exoskeleton. In another word, it will show the advantage of max-plus model over the conventional dynamics models in the control of lower limb exoskeleton. However, both the max-plus algebra approach and the conventional approaches, such as Newton Euler or Lagrange, have pros and cons. In general, the max-plus algebra is an approach to describe the discrete event system, which is suitable for the modelling of the relation between walking phases is the whole gait periods, like sequence and constraint among the events, as well as the gait/phase period analysis. While the conventional approaches are suitable for the continuous system, which build the model to describe the dynamic relation between the angles, speed, and torque in each gait phase. The main aim of this paper is to introduce a new modelling approach for the researchers in this field, with no hint that the conventional approaches are no longer useful.

The paper is organized as follows: first, the preliminary of max-plus is generally introduced. Then the walking process is divided into some discrete events and described in timed event graph. Next, the lower limb exoskeleton system used in this paper is presented. In the following section, the autonomous control approach based on the max-plus algebra is proposed. An experiment combined with FMS (finite state machine) is presented in the next section. And some concluding remarks are given in the end.

\section{Preliminary of Max-Plus Algebra}

Max-plus algebra was proposed in the 1950s for the research of traffic planning and production schedule. In the following decades, Cuninghame-Green [19], Baccelli et al [20], and Heidergott et al [5] made great contribution on it. Some basic rules are introduced as follows.

where $R$ is defined as the set of all real numbers and $R_{\max }$ is defined as the set $R \cup\{-\infty\}$. The operation of $\max$ is represented by $\oplus$. For $a, b \in R_{\max }$, the operation of max-plus algebra is shown as follows:

$$
\begin{aligned}
& a \oplus b=\max (a, b), \\
& a \otimes b=a+b .
\end{aligned}
$$

The operation of plus is represented by $\otimes ;-\infty$ is denoted by $\epsilon$ in max-plus algebra; and 0 is denoted by $e$ in max-plus algebra. There are some examples of max-plus algebra:

$$
\begin{aligned}
& a \oplus \varepsilon=\varepsilon \oplus a=a, \\
& a \otimes \varepsilon=\varepsilon \otimes a=\varepsilon, \\
& 3 \oplus 5=\max (3,5)=5, \\
& e \oplus 5=\max (0,5)=5, \\
& 4 \oplus \varepsilon=\max (4,-\infty)=4, \\
& 6 \otimes 3=6+3=9, \\
& 6 \otimes \varepsilon=6-\infty=\varepsilon .
\end{aligned}
$$

For matrices operation in max-plus algebra, operations $\oplus$ and $\otimes$ are shown as follows:

$$
\begin{aligned}
& (A \oplus B)_{i j}=a_{i j} \oplus b_{i j}, \\
& {[A \otimes B]_{i j}=\oplus_{k=1}^{l} a_{i k} \otimes b_{k j}=\max \left\{a_{i k}+b_{k j}\right\},}
\end{aligned}
$$

where matrices $A=\left(a_{i j}\right), B=\left(b_{i j}\right)$.

$$
\begin{aligned}
& \text { where matrices } A=\left(a_{i j}\right), B=\left(b_{i j}\right) \text {. } \\
& \text { Some examples are shown for } A=\left[\begin{array}{ll}
e & 5 \\
\varepsilon & 2
\end{array}\right] \text { and } \\
& B=\left[\begin{array}{ll}
1 & 8 \\
3 & \varepsilon
\end{array}\right]: \\
& A \oplus B=\left[\begin{array}{ll}
1 & 8 \\
3 & 2
\end{array}\right] \\
& A \otimes B=\left[\begin{array}{ll}
e \otimes 1 \oplus 5 \otimes 3 & e \otimes 8 \oplus 5 \otimes \varepsilon \\
\varepsilon \otimes 1 \oplus 2 \otimes 3 & \varepsilon \otimes 8 \oplus 2 \otimes \varepsilon
\end{array}\right]=\left[\begin{array}{ll}
8 & 8 \\
5 & \varepsilon
\end{array}\right], \\
& B \otimes A=\left[\begin{array}{ll}
1 \otimes e \oplus 8 \otimes \varepsilon & 1 \otimes 5 \oplus 8 \otimes 2 \\
3 \otimes e \oplus \varepsilon \otimes \varepsilon & 3 \otimes 5 \oplus \varepsilon \otimes 2
\end{array}\right]=\left[\begin{array}{ll}
1 & 10 \\
3 & 8
\end{array}\right]
\end{aligned}
$$

It should be noticed that $A \otimes B$ is not equal to $B \otimes A$, which is similar with the quality of the matrix operation in conventional linear algebra. 
The identity matrix in max-plus algebra and its operation example are as follows:

$$
\begin{aligned}
I & =\left[\begin{array}{llll}
e & \varepsilon & \cdots & \varepsilon \\
\varepsilon & e & \cdots & \varepsilon \\
\vdots & \vdots & & \vdots \\
\varepsilon & \varepsilon & \cdots & \varepsilon
\end{array}\right], \\
B \oplus I & =\left[\begin{array}{ll}
1 & 8 \\
3 & e
\end{array}\right], \\
A \otimes I & =\left[\begin{array}{ll}
e & 5 \\
\varepsilon & 2
\end{array}\right]=A .
\end{aligned}
$$

\section{Walking Process in Timed Event Graph}

In this section, a timed event graph is used to describe the walking process. In the timed event graph, vertical bar denotes the event. As shown in Figure 1, the bar $q_{1}$ denotes left foot lifting and $q_{2}$ denotes left foot falling. While the bars $q_{3}$ and $q_{4}$ denote the event of right foot lifting and falling, respectively. In normal walking process, the timed event loop should be $q_{1} q_{2} q_{3} q_{4} q_{1}$.

The circle denotes the process, and the dot in the circle denotes that the corresponding process is in progress. As shown in Figure 1, the circle $a_{21}$ and $a_{12}$ denote the time of left foot lifting off and falling down the ground, respectively; $a_{43}$ and $a_{34}$ denote the time of right foot lifting off and falling down the ground, respectively; and $a_{32}$ and $a_{14}$ denote the time of foot beginning to lift after the swaying contralateral foot falls down the ground, which is to ensure that the contralateral foot stand firmly.

As shown in Figure 1, to assure the safety in the walking process, foot lifting can only happen after both feet fall on the ground firmly. So $q_{1}$ must wait the finish of both $a_{12}$ and $a_{14} ; q_{3}$ must wait the finish of both $a_{32}$ and $a_{34}$, in which a max function between $a_{12}$ and $a_{14}, a_{32}$, and $a_{34}$ is needed. Such logical sequence could not be described in conventional model methods, which makes it difficult to avoid the danger in the walking control based on those conventional models. However, the model based on max-plus could describe such logical sequence and safety condition. As a result, the theoretical analysing approach of max-plus could also provide mathematic tools for the control of exoskeleton. To sum up, some assumptions in the walking process are given as follows.

Assumption 1. $q_{1}$ cannot begin unless both $a_{12}$ and $a_{14}$ are over.

Assumption 2. $q_{3}$ cannot begin unless both $a_{32}$ and $a_{34}$ are over.

Assumption 3. $q_{2}$ cannot begin unless $a_{21}$ is over.

Assumption 4. $q_{4}$ cannot begin unless $a_{43}$ is over.

\section{Lower Limb Exoskeleton System}

A multisensor hip-driven exoskeleton system is used in this paper, as shown in Figure 2. Angle sensors are mounted on thighs to measure the hip joint angle. Plantar pressure sensors are mounted under the feet. Although there are 8 sample pints, only the one under the heel is used in this paper. And the one under the toe is used only for reference. If the foot strikes the ground, the output of plantar pressure sensor is 1 . While the foot is off the ground, the output of plantar pressure sensor is 0 . So, as shown in Figure 3, the time for leg/foot lifting up, which is denoted by $a_{21}$ and $a_{43}$, begins from the 0 state of plantar pressure sensor and ends at the time of the hip angle sensor reaches the maximum value. While the time for leg/foot falling down, which is denoted by $a_{12}$ and $a_{34}$, begins from the maximum value of hip angle sensor to the 1 state of plantar pressure sensor. $a_{14}$ and $a_{32}$ begin from the maximum value of hip angle sensor to the 0 state of contralateral plantar pressure sensor.

Besides, two pairs of spring-pressure sensors are amounted at the front and back of the thigh between the exoskeleton and the human body to detect the intention of human walking. When the output of plantar pressure sensor is 1, which denotes that the foot is on the ground, if the output of front spring-pressure sensor is higher than the back one, it means that the exoskeleton wearer is intending to lift his leg. Then the microcomputer will give the motor an order to drive the exoskeleton rotating forward. On the contrary, when the output of plantar pressure sensor is 0 , which denotes that the foot is off the ground, if the output of front spring-pressure sensor is lower than the back one, it means that the exoskeleton wearer is intending to fall his leg. Then the microcomputer will give the motor an order to drive the exoskeleton rotating backward. By the way, as the comparison of pressure is easily affected by the vibration of springs, a threshold must be set in the judgment of wearer's motion intention to eliminate the interference.

\section{Walking Process Modeling via Max- Plus Algebra}

As mentioned earlier, the walking process could be considered as four discrete events, the lifting and falling of two legs, which is denoted as $q_{i}, i=1,2,3,4$. The time of each event could be modelled as the discrete event state equation in

$$
\begin{aligned}
& x_{1}(k+1)=\max \left\{x_{2}(k)+a_{12}, x_{4}(k)+a_{14}\right\}, \\
& x_{2}(k+1)=x_{1}(k+1)+a_{21}, \\
& x_{3}(k+1)=\max \left\{x_{2}(k+1)+a_{32}, x_{4}(k)+a_{34}\right\}, \\
& x_{4}(k+1)=x_{3}(k+1)+a_{43},
\end{aligned}
$$

where $x_{i}(k)$ represents the beginning moment of the $k$ th occurrence of event $q_{i}$.

The max operation the $x_{1}(k+1)$ and $x_{3}(k+1)$ ensure the following constraints: the foot lifting must occur after it is on the ground and the contralateral foot supports firmly.

Equation (6) can also be expressed as 


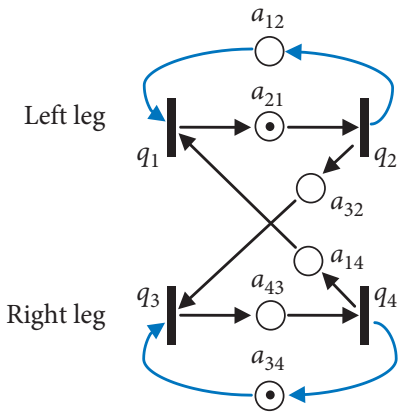

Figure 1: Timed event graph of walking.

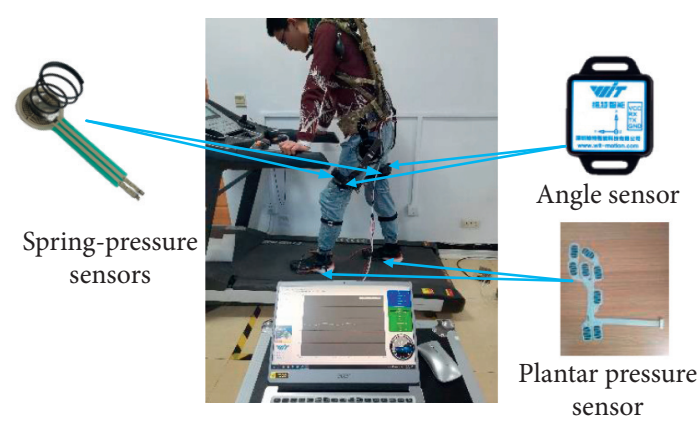

FIgURE 2: Exoskeleton system.

$$
x(k+1)=A_{0} x(k+1) \oplus A_{1} x(k),
$$

where

$$
\begin{aligned}
& A_{0}=\left[\begin{array}{cccc}
\varepsilon & \varepsilon & \varepsilon & \varepsilon \\
a_{21} & \varepsilon & \varepsilon & \varepsilon \\
\varepsilon & a_{32} & \varepsilon & \varepsilon \\
\varepsilon & \varepsilon & a_{43} & \varepsilon
\end{array}\right], \\
& A_{1}=\left[\begin{array}{cccc}
\mathcal{E} & a_{12} & \varepsilon & a_{14} \\
\varepsilon & \varepsilon & \varepsilon & \varepsilon \\
\varepsilon & \varepsilon & \varepsilon & a_{34} \\
\varepsilon & \varepsilon & \varepsilon & \varepsilon
\end{array}\right], \\
& A_{1}=\left[\begin{array}{llll}
\mathcal{E} & a_{12} & \varepsilon & a_{14} \\
\varepsilon & \varepsilon & \varepsilon & \varepsilon \\
\varepsilon & \varepsilon & \varepsilon & a_{34} \\
\varepsilon & \varepsilon & \varepsilon & \varepsilon
\end{array}\right] .
\end{aligned}
$$

Iterate equation (7):

$x(k+1)=A_{0}^{4} x(k+1) \oplus\left(A_{0}^{3} \oplus A_{0}^{2} \oplus A_{0} \oplus I\right) A_{1} x(k)$.

After the 4 th iterative calculation, there will be $A_{0}^{4}=\varepsilon$. So

$$
x(k+1)=\left(A_{0}^{3} \oplus A_{0}^{2} \oplus A_{0} \oplus I\right) A_{1} x(k):=A x(k),
$$

where $I$ is the identity matrices and

$$
A=\left[\begin{array}{cccc}
\varepsilon & a_{12} & \varepsilon & a_{14} \\
\varepsilon & a_{12} a_{21} & \varepsilon & a_{14} a_{21} \\
\varepsilon & a_{12} a_{21} a_{32} & \varepsilon & a_{14} a_{21} a_{32} \oplus a_{34} \\
\varepsilon & a_{12} a_{21} a_{32} a_{43} & \varepsilon & a_{14} a_{21} a_{32} a_{43} \oplus a_{34} a_{43}
\end{array}\right] .
$$

According to the literature studies $[21,22]$, if there exist a positive number $\lambda$ and a positive vector $v$ that $A v=\lambda v$, then $\lambda$ is an eigenvalue of $A$ and $v$ is its eigenvector of $A$ relative to $\lambda$. And each square max-plus matrix has at least one eigen value.

In the control of lower limb exoskeleton, eigenvalue $\lambda$ denotes the time length of a stride period. The steady state of $\lambda$ means the regularity and moderate walking speed for the exoskeleton wearers. If the motion behaviour of the exoskeleton is properly coordinated in the walking, the control process, eigenvalue $\lambda$, should be kept stable. Otherwise, there will be disturbance in body balance or uneven walking speed. The periodic steady state analyses and the disturbance estimation of lower limb exoskeleton based on the max-plus algebra have been presented in our former paper [18], where

$$
\lambda=a_{12} a_{21} \oplus a_{14} a_{43} a_{32} a_{21} \oplus a_{34} a_{43} .
$$

In this paper, the further research on the autonomous control of walking phases will be discussed in the following section, which ensures the periodic performance and safety of lower limb exoskeleton. 


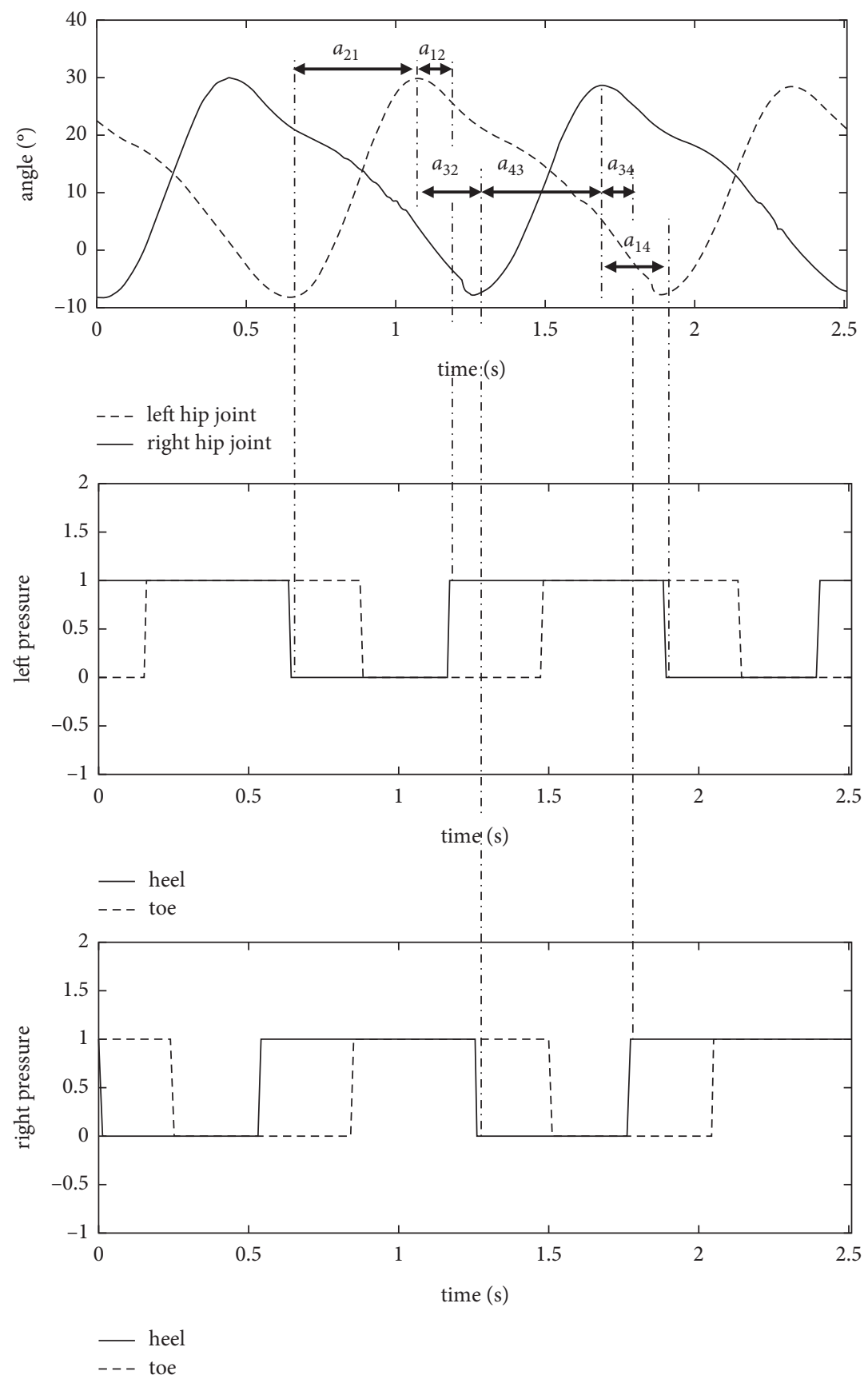

Figure 3: The time of each walking state/phase.

\section{Autonomous Control}

In the practical application, exoskeleton wearers need to change the walking stride or speed, which can be considered as a system with autonomous control. Figure 4 is the timed event graph model of the lower limb exoskeleton with autonomous control, in which the external input $U$ is the switch to control the start of each motion phase in the process of walking movement.

Such a lower limb exoskeleton system can be described by the following discrete event state equations:

$$
\begin{aligned}
& x_{1}(k+1)=\max \left\{x_{2}(k)+a_{12}, x_{4}(k)+a_{14}, u_{1}(k+1)\right\}, \\
& x_{2}(k+1)=\max \left\{x_{1}(k+1)+a_{21}, u_{2}(k+1)\right\}, \\
& x_{3}(k+1)=\max \left\{x_{2}(k+1)+a_{32}, x_{4}(k)+a_{34}, u_{3}(k+1)\right\}, \\
& x_{4}(k+1)=\max \left\{x_{3}(k+1)+a_{43}, u_{4}(k+1)\right\} .
\end{aligned}
$$

For simplicity of presentation, the system is denoted as $H_{u}$. System $H_{u}$ can be expressed as the following max-plus linear equation: 
$x(k+1)=A_{0} x(k+1) \oplus A_{1} x(k) \oplus B u(k+1)$,

where $u(\mathrm{k}) \in R$ is the input vector; $A_{0}$ and $A_{1}$ are the same as the matrices in equation (7); and $B=[0 \in \epsilon \in]^{\mathrm{T}}$.

Iterate equation (14):

$$
\begin{aligned}
x(k+1)= & A_{0}^{4} x(k+1) \oplus\left(A_{0}^{3} \oplus A_{0}^{2} \oplus A_{0} \oplus I\right) A_{1} x(k) \\
& \oplus\left(A_{0}^{3} \oplus A_{0}^{2} \oplus A_{0} \oplus I\right) B u(k+1) .
\end{aligned}
$$

As $A_{0}^{4}=\varepsilon$, so the equation above can be written as

$$
\begin{aligned}
x(k+1) & =\left(A_{0}^{3} \oplus A_{0}^{2} \oplus A_{0} \oplus I\right) A_{1} x(k) \oplus\left(A_{0}^{3} \oplus A_{0}^{2} \oplus A_{0} \oplus I\right) B u(k+1) \\
& =\left[\begin{array}{cccc}
\varepsilon & a_{12} & \varepsilon & a_{14} \\
\varepsilon & a_{12} a_{21} & \varepsilon & a_{14} a_{21} \\
\varepsilon & a_{12} a_{21} a_{32} & \varepsilon & a_{14} a_{21} a_{32} \oplus a_{34} \\
\varepsilon & a_{12} a_{21} a_{32} a_{43} & \varepsilon & a_{14} a_{21} a_{32} a_{43} \oplus a_{34} a_{43}
\end{array}\right] x(k) \oplus\left[\begin{array}{c}
0 \\
a_{21} \\
a_{21} a_{32} \\
a_{21} a_{32} a_{43}
\end{array}\right] u(k) .
\end{aligned}
$$

By setting the input sequence $\{u(k)\}$, autonomous control of lower limb exoskeleton system can be realized. The length of the input sequence $k$ determines the number of the walking motion cycles, and the component of vector $u(k)$ determines the start time of each motion in the $k$ th walking cycle.

Example 1. The parameters of a nonautonomous lower limb exoskeleton system $H_{u}$, are shown as follows:

$$
\begin{aligned}
& a_{14}=0.6, \\
& a_{43}=0.9, \\
& a_{32}=0.6, \\
& a_{21}=0.9, \\
& a_{12}=0.5, \\
& a_{34}=0.5 .
\end{aligned}
$$

The initial conditions are $x(0)=\left(\begin{array}{llll}0 & 0 & 0 & 0\end{array}\right)^{\mathrm{T}}$. According equations (10) and (12), $\lambda=3$. Take the control of start time of each step as example. The control sequences are $\left\{u_{1}(1)=0\right.$, $\left.u_{1}(2)=3, u_{1}(3)=7\right\}$. The walking process and the corresponding time are shown as follows:

$$
\begin{aligned}
& x(1)=\left[\begin{array}{cccc}
\varepsilon & 0.5 & \varepsilon & 0.6 \\
\varepsilon & 1.4 & \varepsilon & 1.5 \\
\varepsilon & 2 & \varepsilon & 2.1 \\
\varepsilon & 2.9 & \varepsilon & 3
\end{array}\right] x(0) \oplus\left[\begin{array}{c}
0 \\
0.9 \\
1.5 \\
2.4
\end{array}\right] u(1)=\left[\begin{array}{c}
0.6 \\
1.5 \\
2.1 \\
3
\end{array}\right], \\
& x(2)=\left[\begin{array}{cccc}
\varepsilon & 0.5 & \varepsilon & 0.6 \\
\varepsilon & 1.4 & \varepsilon & 1.5 \\
\varepsilon & 2 & \varepsilon & 2.1 \\
\varepsilon & 2.9 & \varepsilon & 3
\end{array}\right] x(1) \oplus\left[\begin{array}{c}
0 \\
0.9 \\
1.5 \\
2.4
\end{array}\right] u(2)=\left[\begin{array}{c}
3.6 \\
4.5 \\
5.1 \\
6
\end{array}\right] \text {, } \\
& x(3)=\left[\begin{array}{cccc}
\varepsilon & 0.5 & \varepsilon & 0.6 \\
\varepsilon & 1.4 & \varepsilon & 1.5 \\
\varepsilon & 2 & \varepsilon & 2.1 \\
\varepsilon & 2.9 & \varepsilon & 3
\end{array}\right] x(2) \oplus\left[\begin{array}{c}
0 \\
0.9 \\
1.5 \\
2.4
\end{array}\right] u(3)=\left[\begin{array}{c}
7 \\
8.5 \\
9.1 \\
10
\end{array}\right] \text {. }
\end{aligned}
$$

The period between $x(2)$ and $x(1)$ is 3 , which is equal to $\lambda$. However, the period between $x(3)$ and $x(2)$ is not 3 , so to ensure the stability and coordination of the walking process, a state feedback control could be added on the system as follows:

$$
u(k+1)=a x_{4}(k)
$$

The state equation of the closed-loop feedback system is

$$
x(k+1)=D x(k)
$$

where

$$
D=\left[\begin{array}{cccc}
\varepsilon & a_{12} & \varepsilon & t \\
\varepsilon & a_{12} a_{21} & \varepsilon & t a_{21} \\
\varepsilon & a_{12} a_{21} a_{32} & \varepsilon & t a_{21} a_{32} \oplus a_{34} \\
\varepsilon & a_{12} a_{21} a_{32} a_{43} & \varepsilon & t a_{21} a_{32} a_{43} \oplus a_{34} a_{43}
\end{array}\right],
$$

and $t-a_{14} \oplus a$. The precedence graph $G(D)$ of matrix $D$ is shown in Figure 5.

As shown in Figure 5, the period of closed-loop system is

$$
\tilde{\lambda}=a_{12} a_{21} \oplus t a_{43} a_{32} a_{21} \oplus a_{34} a_{43} .
$$

So, the state feedback can adjust the period of lower limb exoskeleton system to regulate the walking speed. The robustness of periodic steady-state performance with respect to feedback parameter $a$ is discussed here. And the regulating range of feedback parameter $a$, which keeps stable steady-state performance, will be determined.

Lemma 1. The walking period $\lambda$ of lower limb exoskeleton system $H$ is robust with respect to feedback parameter $a$, in which the regulating range of feedback parameter $a$ is

$$
\begin{aligned}
a & \leq a_{14} \\
\text { or } a_{14} & <a \leq \max \left(a_{12}-a_{32}-a_{43}, a_{34}-a_{21}-a_{32}\right) \text {. }
\end{aligned}
$$

Proof. When $a \leq a_{14}, t=a_{14} \oplus a=a_{14}$. So, $\tilde{\lambda}=\lambda$; when $a_{14}<a \leq \max \left(a_{12}-a_{32}-a_{43}, a_{34}-a_{21}-a_{32}\right)$,

$$
\begin{aligned}
t a_{21} a_{32} a_{43} & =a a_{21} a_{32} a_{43} \leq \max \left(a_{12}+a_{21}, a_{34}+a_{43}\right) \\
& =a_{12} a_{21} \oplus a_{34} a_{43}, \\
a_{14} a_{21} a_{32} a_{43} & <a a_{21} a_{32} a_{43} \leq a_{12} a_{21} \oplus a_{34} a_{43} .
\end{aligned}
$$




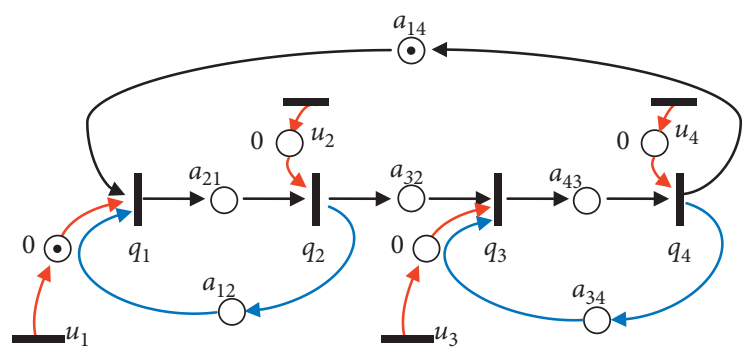

Figure 4: Timed event graph of autonomous-controlled lower limb walking.

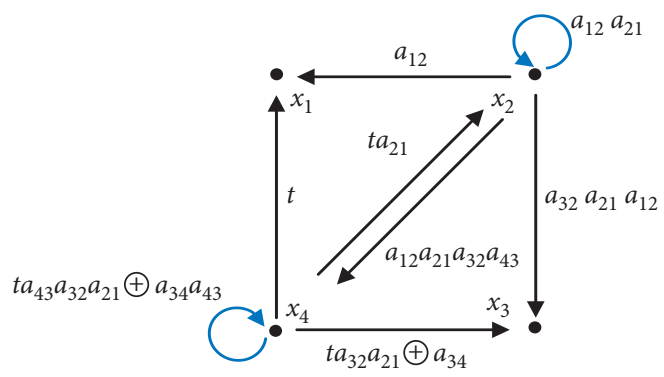

Figure 5: Precedence graph $G(D)$ of matrix $D$.

So,

$$
\tilde{\lambda}=\lambda=a_{12} a_{21} \oplus a_{34} a_{43} .
$$

Example 2. Add state feedback on the lower limb exoskeleton system $H_{u}$ shown in the Example 1. As it can be shown that

$$
a_{14}=0.6>-1=\max \left(a_{12}-a_{32}-a_{43}, a_{34}-a_{21}-a_{32}\right) \text {. }
$$

So, in order to keep the steady-state performance stable, the regulating range of feedback parameter $a$ is $a \leq a_{14}=0.6$. For example, if we set $a=0.5$, then the state matrix of the closed system (19) is the same as Example 1, as shown below:

$$
D=\left[\begin{array}{cccc}
\varepsilon & 0.5 & \varepsilon & 0.6 \\
\varepsilon & 1.4 & \varepsilon & 1.5 \\
\varepsilon & 2 & \varepsilon & 2.1 \\
\varepsilon & 2.9 & \varepsilon & 3
\end{array}\right]=A,
$$

where $D$ is the state matrix of the closed system.

And the period of the system remains unchanged, which is 3 . If we set $a=0.8$, then the state matrix of the closed system (27) will be

$$
D=\left[\begin{array}{cccc}
\varepsilon & 0.5 & \varepsilon & 0.8 \\
\varepsilon & 1.4 & \varepsilon & 1.7 \\
\varepsilon & 2 & \varepsilon & 2.3 \\
\varepsilon & 2.9 & \varepsilon & 3.2
\end{array}\right] .
$$

Then the system period is changed into 3.2 due to the regulating effect of feedback parameter $a$.

\section{Finite State Machine Control and Max-Plus Algebra Model}

A finite state machine (FSM) is an event-driven control approach for the discrete system, in which an FSM makes a transition from one state to another, if the condition defining the change is true $[22,23]$. In the motion control of lower limb exoskeleton via FSM, the transmission has four operating states or the walking phases: left foot lifting, left foot falling, right foot lifting, and right foot falling. To control the joint-driven motors on the exoskeleton, the FSM can make a transition from one walking state to another according to the trigger signal from the exoskeleton wearer.

However, FMS is a control technique without effective fundamental of mathematic model. As a result, the periodic steady state of FSM cannot be analysed, nor is the period control. While the max-plus algebra can provide effective tools for FSM. In this paper, an FSM controller based on max-plus algebra model is proposed. The trigger signal comes from both the exoskeleton wearer and the control signals of max-plus algebra model. So the start time of each phase/state in the walking process can be regulated and a steady walking stride can be obtained. And the max operation in max-plus algebra and the "AND" operation of trigger signals in FSM can guarantee the safety in walking. The desired walking speed might be regulated via the parameters $a_{i j}$ and $U_{m}$ in the max-plus algebra model. $a_{i j}$ is transferred into FSM as the scale factor of driving power $U_{d i}$ on the motors. $U_{m}$ is transferred into FSM as a necessary state transition condition, which plays the role of keeping safe and constant walking speed. The state transition conditions of FSM are shown in Table 1. In Table $1, P_{f}$ denotes the pressure between the front of thigh and the exoskeleton, $P_{b}$ denotes the pressure 
TABLE 1: The state transition conditions of FSM.

\begin{tabular}{lcccc}
\hline Condition no. $^{*}$ & Motion & $U_{m}$ & $P_{f}-P_{b}$ & $P_{h}$ \\
\hline $\mathrm{C}_{1}$ & Left foot lifts & $u_{1}=1$ & $>\Delta P$ & $1 \longrightarrow 0$ \\
$\mathrm{C}_{2}$ & Left foot falls & $u_{2}=1$ & $<\Delta P$ & 0 \\
$\mathrm{C}_{3}$ & Right foot lifts & $u_{3}=1$ & $>\Delta P$ & 1 \\
$\mathrm{C}_{4}$ & Right foot falls & $u_{4}=1$ & $<\Delta P$ & $1 \longrightarrow 0$ \\
\hline
\end{tabular}

*The index of denoting the left/right foot/leg is omitted.

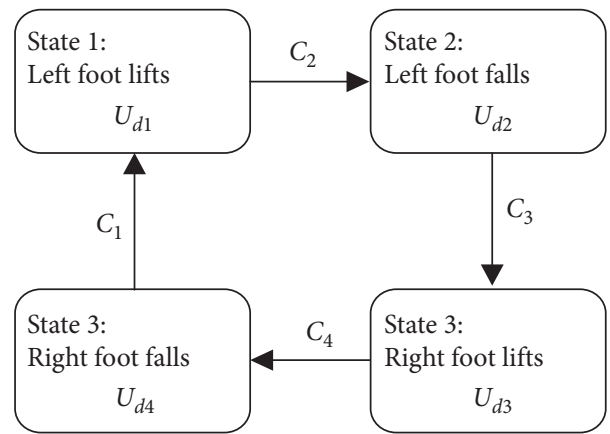

(a)
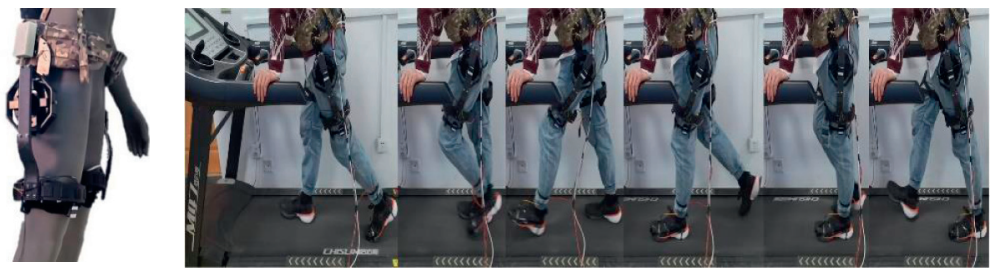

(b) (c)

Figure 6: Experiment based on FSM. (a) State flow of FSM. (b) Exoskeleton. (c) Experiment.

between the back of thigh and the exoskeleton, $\Delta P$ is a preset threshold to eliminate the influence caused by irregular vibration in the walking, $P_{h}$ denotes the heel pressure, and $P_{t}$ denotes toe pressure.

The state flow and experiment of FSM are shown in Figure 6. A set of hip joint exoskeleton system is used in the experiment. The joint is driven by a plat brushless DC servo motor, and the motor model is Innfos qdd-pro-nu80, the rated torque is $103 \mathrm{~nm}$, and the maximum torque is $289 \mathrm{~nm}$. The sensors on the exoskeleton include angular sensors, plantar pressure sensors, and spring-pressure sensors. The experiment is carried on a treadmill.

In the experiment, the exoskeleton operates consistent with the speed of the treadmill. The experiment shows that the function of max-plus algebra embedded in the control program can effectively keep steady walking and avoid the mistriggering in FSM caused by the interfered sensor signals. The time constraint condition based on max-plus algebra can prevent the leg lift unless the contralateral foot is in firm support phase. And the recorded off-line data of the repeated walking phases show that the gait/phase periods are also kept stable.

\section{Conclusion}

In this paper, the periodic steady state, disturbance estimation, and autonomous control of the lower limb exoskeleton system are studied. Based on the timed event graph and the max-plus algebra method, the mathematical model of the lower limb exoskeleton is established. And the time disturbance in the system is discussed. An autonomous control strategy is proposed. It provided useful analysing tools for the modelling and safe control of the exoskeleton.
The model based on max-plus can only describe the logical timed sequence of the whole walking process, which is suitable for the application of FSM (finite state machine), while, as normal human gait is not a statically stable system, but rather a dynamically stable system, the conventional model based on the Newton Euler method or the Lagrange method could provide the physical information of each motion phase in the walking process, which is suitable for the application of other control approaches to obtain dynamic stability. Both modelling approaches have its merits and demerits. This paper discusses the high level control of exoskeleton action based on max-plus algebra, while the "Newton Euler method and the Lagrange method" are usually used in trajectory planning and feedback gain design. The two approaches are useful in different level of the exoskeleton control. So, the research on the dual-model exoskeleton system might be one important future works.

Additionally, some other control approaches, such as model-free control [24, 25], sliding mode control [26], and ILC (iterative learning control), [27] and reinforcement learning $[28,29]$ might be tried on the base of max-plus algebra model too. Moreover, the research combined with lower limb motion intention recognition and biomedical engineering are also necessary [30-33].

\section{Data Availability}

The data used to support the findings of this study are available from the corresponding author upon request.

\section{Conflicts of Interest}

The authors declare that they have no conflicts of interest. 


\section{Authors' Contributions}

Cuifeng Qin contributed to conceptualization, formal analysis, methodology, and original draft preparation and Zuojun Liu contributed to methodology, validation, review, editing, and supervision. All authors have read and agreed to the published version of the manuscript.

\section{Acknowledgments}

The authors appreciate Professor Yuegang Tao at Hebei University of Technology in China for his help in the field of max-plus algebra. This research was funded by the "Program of Introduction Talent of Hebei Province," "Science Program of Tianjin (19YFHBQY00040)," and "Chinese NSF, grant no. 61703135."

\section{References}

[1] G. Orekhov, Y. Fang, J. Luque, and Z. F. Lerner, "Ankle exoskeleton assistance can improve over-ground walking economy in individuals with cerebral palsy," IEEE Transactions on Neural Systems and Rehabilitation Engineering, vol. 28, no. 2, pp. 461-467, 2020.

[2] A. R. Dawson-Elli and P. G. Adamczyk, "Design and validation of a lower-limb haptic rehabilitation robot," IEEE Transactions on Neural Systems and Rehabilitation Engineering, vol. 28, no. 7, pp. 1584-1594, 2020.

[3] G. Bao, L. Pan, H. Fang et al., "Academic review and perspectives on robotic exoskeletons," IEEE Transactions on Neural Systems and Rehabilitation Engineering, vol. 27, no. 11, pp. 2294-2304, 2019.

[4] W. Huo, S. Mohammed, and Y. Amirat, "Impedance reduction control of a knee joint human-exoskeleton system," IEEE Transactions on Control Systems Technology, vol. 27, no. 6, pp. 2541-2556, 2019.

[5] B. Heidergott, G. J. Olsder, and J. van der Woude, Max-Plus at Work: Modeling and Analysis of Synchronized Systems, Princeton University Press, Princeton, NJ, USA, 2006.

[6] S. M. Campbell, C. P. Diduch, and J. W. Sensinger, "Autonomous assistance-as-needed control of a lower limb exoskeleton with guaranteed stability," IEEE Access, vol. 8, pp. 51168-51178, 2020.

[7] Y. Ma, X. Wu, C. Wang, Z. Yi, and G. Liang, "Gait phase classification and assist torque prediction for a lower limb exoskeleton system using kernel recursive least-squares method," Sensors, vol. 19, no. 24, p. 5449, 2019.

[8] M. Li, F. Zhadeng, S. Qiu, X. Wang, F. Chen, and F. Chen, "Towards online estimation of human joint muscular torque with a lower limb exoskeleton robot," Applied Sciences, vol. 8, no. 9, p. 1610, 2018.

[9] Z. Li, K. ZhaoRen, and C. Deng, "Human-cooperative control design of a walking exoskeleton for body weight support," IEEE Transactions on Industrial Informatics, vol. 16, no. 5, pp. 2985-2996, 2020.

[10] B. Lim, J. Lee, and J. Jang, "Delayed output feedback control for gait assistance with a robotic hip exoskeleton," IEEE Transactions on Robotics, vol. 35, no. 4, pp. 1055-1062, 2019.

[11] R. Nasiri, M. Rayati, and M. N. Ahmadabadi, "Feedback from mono-articular muscles is sufficient for exoskeleton torque adaptation," IEEE Transactions on Neural Systems and Rehabilitation Engineering, vol. 27, no. 10, pp. 2097-2106, 2019.
[12] Q. Zhao, "A remark on inseparability of min-max systems," IEEE Transactions on Automatic Control, vol. 49, no. 6, pp. 967-970, 2004.

[13] C. Wang, Y. Tao, P. Yang, and X. Liu, "Dimension reduction and feedback stabilization for max-plus linear systems and applications in VLSI array processors," IEEE Transactions on Automatic Control, vol. 62, no. 12, pp. 6353-6368, 2017.

[14] J. P. Comet, "Application of max-plus algebra to biological sequence comparisons," Theoretical Computer Science, vol. 293, no. 1, pp. 189-217, 2003.

[15] R. M. P. Goverde, "Railway timetable stability analysis using max-plus system theory," Transportation Research Part B: Methodolo-Gical.vol. 41, no. 2, pp. 179-201, 2007.

[16] G. A. D. Lopes, T. J. J. van den Boom, B. De Schutter, and R. Babuka, "Modeling and control of legged locomotion via switching max-plus models," IEEE Transactions on Robotics, vol. 30, no. 3, pp. 652-665, 2014.

[17] Y. Tao, C. Wang, X. Wang, and P. Yang, "Period steady-state and autonomous control of the upper limb transfer system of rehabilitation robots," Control Theory \& Applications, vol. 35, no. 1, pp. 103-109, 2018.

[18] C. Qin, Z. Liu, and J. Zhang, "Periodic steady state analysis of lower limb rehabilitation robot based on max-plus algebra," in Proceedings of the 5th International Conference on Robotics and Automation Sciences (ICRAS), vol. 07, pp. 33-37, IEEE, Wuhan, China, June 2021.

[19] R. A. Cuninghame-Green, Minimax Algebra, Springer-Verlag, Berlin, Germany, 1979.

[20] F. Baccelli, G. Cohen, G. J. Olsder, and J. -P. Quadrat, Synchronization and Linearity: An Algebra for Discrete Event Systems, John Wiley and Sons, New York, NY, USA, 1992.

[21] B. de Schutter and B. de Moor, "A note on the characteristic equation in the max-plus algebra," Linear Algebra and its Applications, vol. 261, no. 1, pp. 237-250, 1997.

[22] G. J. Olsder and C. Roos, "Cramer and cayley-hamilton in the max algebra," Linear Algebra and its Applications, vol. 101, no. 1, pp. 87-108, 1988.

[23] P. T. Chinimilli, S. Sorkhabadi, and W. Zhang, "Assessment of human dynamic gait stability with a lower extremity assistive device," IEEE Transactions on Neural Systems and Rehabilitation Engineering, vol. 28, no. 3, pp. 669-678, 2020.

[24] X. Zhao, Z. Liu, B. Gou, and P. Yang, "Analysis of slope motion control strategy of lower limb prostheses," Control and Decision, vol. 34, no. 6, pp. 1160-1168, 2019.

[25] S. Han, H. Wang, and Y. Tian, "A linear discrete-time extended state observer-based intelligent PD controller for a 12 DOFs lower limb exoskeleton LLE-RePA," Mechanical Systems and Signal Processing, vol. 138, Article ID 106547, 2020.

[26] S. Ahmed, H. Wang, and Y. Tian, "Adaptive high-order terminal sliding mode control based on time delay estimation for the robotic manipulators with backlash hysteresis," IEEE Transactions on Systems, Man, and Cybernetics: Systems, vol. 51, no. 2, pp. 1128-1137, 2021.

[27] L. Blanken and T. Oomen, "Multivariable iterative learning control design procedures: from decentralized to centralized, illustrated on an industrial printer," IEEE Transactions on Control Systems Technology, vol. 28, no. 4, pp. 1534-1541, 2020.

[28] W. Bai, T. Li, and S. Tong, "NN reinforcement learning adaptive control for a class of nonstrict-feedback discretetime systems," IEEE Transaction on Cybernetics, vol. 50, no. 11, pp. 4573-4584, 2020.

[29] W. Bai, Q. Zhou, T. Li, and H. Li, "Adaptive reinforcement learning neural network control for uncertain nonlinear 
system with input saturation," IEEE Transaction on Cybernetics, vol. 50, no. 8, pp. 3433-3443, 2020.

[30] Z. Liu, W. Lin, Y. Geng, and P. Yeng, "Intent pattern recognition of lower-limb motion based on mechanical sensors," IEEE/CAA Journal of Automatica Sinica, vol. 4, no. 4, pp. 651-660, 2017.

[31] W. Huo, S. Mohammed, J. C. Moreno, and Y. Amirat, "Lower limb wearable robots for assistance and rehabilitation: a state of the art," IEEE System Journal, vol. 10, no. 3, pp. 1068-1081, 2016.

[32] B. Hwang, B. Oh, and D. Jeon, "An optimal method of training the specific lower limb muscle group using an exoskeletal robot," IEEE Transactions on Neural Systems and Rehabilitation Engineering, vol. 26, no. 4, pp. 830-838, 2018.

[33] Z. Liu, X. Gao, X. Zhao, and L. Chen, "The research on running gait recognition and motion control strategy for lower limb prosthesis wearer," Chinese Journal of Scientific Instrument, vol. 39, no. 7, pp. 74-82, 2018. 